\title{
Mean platelet volume predicts chemotherapy response and prognosis in patients with unresectable gastric cancer
}

\author{
LIAN LIAN ${ }^{1,2^{*}}$, YOU-YOU XIA ${ }^{3,4 *}$, CHONG ZHOU $^{5}$, XIAO-MING SHEN ${ }^{2}$, XIANG-LI LI ${ }^{6}$, \\ SHU-GUANG HAN ${ }^{6}$, YAN ZHENG ${ }^{2}$, FEI-RAN GONG ${ }^{7}$, MIN TAO $^{1,8-10}$ and WEI LI ${ }^{1,8,10}$ \\ ${ }^{1}$ Department of Oncology, The First Affiliated Hospital of Soochow University, Suzhou, Jiangsu 215006; \\ ${ }^{2}$ Department of Oncology, Suzhou Xiangcheng People's Hospital, Suzhou, Jiangsu 215131; ${ }^{3}$ Department of Radiation Oncology, \\ Lianyungang First People's Hospital, Lianyungang, Jiangsu 222002; ${ }^{4}$ The Fourth Clinical Medical College, \\ Nanjing Medical University, Nanjing, Jiangsu 210029; ${ }^{5}$ Department of Radiation Oncology, Xuzhou Central Hospital, \\ Xuzhou, Jiangsu 221009; ${ }^{6}$ Department of General Surgery, Suzhou Xiangcheng People's Hospital, Suzhou, Jiangsu 215133; \\ ${ }^{7}$ Department of Hematology, The First Affiliated Hospital of Soochow University, Suzhou, Jiangsu 215006; \\ ${ }^{8}$ Jiangsu Institute of Clinical Immunology; ${ }^{9}$ Institute of Medical Biotechnology, Soochow University, Suzhou, Jiangsu 215007; \\ ${ }^{10}$ PREMED Key Laboratory for Precision Medicine, Soochow University, Suzhou, Jiangsu 215021, P.R. China
}

Received August 14, 2015; Accepted October 2, 2015

DOI: $10.3892 / \mathrm{ol} .2015 .3784$

\begin{abstract}
Gastric cancer is the fourth most frequent cancer and the second cause of cancer-related mortalities worldwide. Platelets play an important and multifaceted role in cancer progression. Elevated mean platelet volume (MPV) detected in peripheral blood has been identified in various types of cancer. In the present study, we investigated the application value of MPV in the prediction of chemotherapy response and prognosis in patients with unresectable gastric cancer. A total of 128 patients with unresectable gastric cancer were included and divided according to the median values of baseline MPV (low MPV: $<11.65$ or high MPV: $\geq 11.65$ ). A low baseline MPV level was correlated with reduced metastasis. The results showed that patients with a low baseline level of MPV improved response to chemotherapy. Changes in MPV were associated with therapeutic efficacy. Patients who remained in or were transferred into the low MPV level subgroup following first-line chemotherapy had improved response, compared to those remaining in or being transferred into the high MPV level group. The patients with a higher baseline MPV had decreased progression-free and overall survival ratios. Univariate and multivariate analyses revealed that baseline MPV was a prog-
\end{abstract}

Correspondence to: Professor Min Tao or Dr Wei Li, Department of Oncology, The First Affiliated Hospital of Soochow University, 188 Shizi Street, Suzhou, Jiangsu 215006, P.R. China

E-mail:mtao@medmail.com.cn; taomin@suda.edu.cn

E-mail: liwei10@suda.edu.cn

*Contributed equally

Key words: gastric cancer, mean platelet volume, chemotherapy, prognosis nostic factor affecting progression-free survival. In conclusion, the results showed that MPV measurements can provide important prognostic information for gastric cancer patients.

\section{Introduction}

Gastric cancer is the fourth most common malignant disease worldwide and the second most common cause of mortality from cancer (1). Countries in East Asia have a higher incidence of gastric cancer (i.e., $>40$ cases per 100,000). Data for individual countries have shown that gastric cancer is the most common cancer in Japan and the second most common in China and Korea (2). Although early diagnosis and treatment of gastric cancer can significantly improve prognosis, the 5-year survival rate is only $10-15 \%$ in individuals with advanced disease (3). This poor outcome may be due to the high incidence of serosal invasion, direct invasion into the adjacent organs and early metastasis $(4,5)$. Biomarkers, such as carbohydrate antigen 19-9 and carcinoembryonic antigen are unsatisfactory due to their low sensitivity $(5,6)$. Therefore, identification of novel biomarkers for the diagnosis and follow up of gastric cancer is essential.

Platelets (PLTs) play an important and multifaceted role in cancer progression (7). Firstly, PLTs facilitate metastasis (8). During hematogenous dissemination, the interaction between circulating tumor cells and PLTs is believed to promote tumor cell survival within the circulation (9) and increase the arrest of tumor cell emboli within the microcirculation (10). Secondly, various studies demonstrated that the release of pro-inflammatory cytokines by cancer, such as interleukin (IL)-1, IL-3, and IL-6, promotes the proliferation of megakaryocytes, leading to the gradual establishment of thrombocytosis (11). Considering the close relationship between PLTs and cancer, biomarkers derived from PLTs are important. Elevated mean platelet volume (MPV) detected in peripheral blood has been identified in various types of cancer, including hepatocellular 
carcinoma (12), ovarian cancer (13), colon cancer (14), lung cancer and breast cancer (15), suggesting that PLT-associated markers serve as potential candidates for the diagnosis and follow up of gastric cancer.

In the present study, we investigated whether MPV provided beneficial diagnostic and prognostic information for patients with unresectable gastric cancer.

\section{Materials and methods}

Subjects and inclusion criteria. The study was conducted as a retrospective investigation of gastric cancer patients who had been referred to the First Affiliated Hospital of Soochow University (Suzhou, China) between June 2010 and June 2011. Approval for the study was granted by the Medical Ethics Committees of the First Affiliated Hospital of Soochow University.

In total, 128 inoperable gastric cancer patients were recruited in this study. Of the 128 patients, 53 patients were locally advanced and the remaining 75 patients were relapsed or metastastic. Patient characteristics are detailed in Table I. The mean age of the 128 patients was 68 years (range, 32-82 years). The inclusion criteria were as follows: a) those with histologically or cytologically confirmed recurrent or metastatic gastric cancer; b) age $>18$ years; c) Karnofsky performance status score of $\geq 70$; d) those with a predicted survival of $\geq 3$ months; e) either naive to antitumor treatment or the postoperative adjuvant chemotherapy was performed $\geq 6$ months after the last dose of chemotherapy; f) in case of patients who were scheduled for radiotherapy on the target lesion, radiotherapy was required to have been finished for $\geq 3$ months; g) those with $\geq 1$ measurable lesion [minimum $10 \times 10 \mathrm{~mm}$ on computed tomography (CT) or magnetic resonance imaging]; and $\mathrm{h}$ ) those who met the following laboratory criteria: white blood cells $(\mathrm{WBC}) \geq 4.0 \times 10^{9} / 1$; absolute neutrophil count $\geq 1.5 \times 10^{9} / 1 ;$ PLT $\geq 100 \times 10^{9} / 1$; serum bilirubin $\leq$ upper limit of normal (ULN); alanine aminotransferase, aspartate aminotransferase and alkaline phosphatase $\leq$ ULNx2.5 (if without liver metastasis) or $\leq \mathrm{ULNx} 5$ (if with liver metastasis); urea nitrogen $\leq$ ULNx1.25; and creatinine $\leq$ ULNx1.25.

Blood samples. Blood (5-7 ml) was collected in a sterile

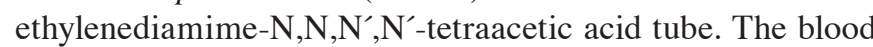
samples were obtained between 06:30 a.m. and 07:30 a.m. to standardize the known impact of circulating hormones (circadian rhythm) on the number and subtype distribution of the various WBC indices. Hematological parameters were analyzed within 30 min after collection using a hematology analyzer (XE2100; Sysmex Corp., Kobe, Japan) and MPV levels were recorded.

Chemotherapy and evaluation. Patients were administered first-line chemotherapy according to the clinical practice guideline for gastric cancer (2006, the first edition) of National Comprehensive Cancer Network. 5-Fluorouracil (5-FU)/leucovorin, 5-FU-based, cisplatin-based, oxaliplatin-based, taxane-based, and irinotecan-based, epirubicin, cisplatin and fluorouracil were recommended. CT scanning was performed for the assessment of response every 2 months and evaluated according to the Response Evaluation Criteria in Solid Tumors 1.1 criteria (16).
Table I. Relationship between MPV and clinicopathological characteristics.

\begin{tabular}{|c|c|c|c|c|c|}
\hline \multirow[b]{2}{*}{$\begin{array}{l}\text { Clinicopathological } \\
\text { characteristics }\end{array}$} & \multirow[b]{2}{*}{ No. } & \multicolumn{2}{|c|}{ MPV } & \multirow[b]{2}{*}{$\chi^{2}$ test } & \multirow[b]{2}{*}{ P-value } \\
\hline & & $\begin{array}{l}\text { High } \\
\text { (no.) }\end{array}$ & $\begin{array}{l}\text { Low } \\
\text { (no.) }\end{array}$ & & \\
\hline Gender & & & & 0.298 & 0.585 \\
\hline Male & 79 & 41 & 38 & & \\
\hline Female & 49 & 23 & 26 & & \\
\hline \multicolumn{6}{|l|}{ Age, years } \\
\hline$<65$ & 72 & 38 & 34 & 0.508 & 0.476 \\
\hline$\geq 65$ & 56 & 26 & 30 & & \\
\hline Tumor size, $\mathrm{cm}$ & & & & 1.276 & 0.259 \\
\hline$<5$ & 86 & 40 & 46 & & \\
\hline$\geq 5$ & 42 & 24 & 18 & & \\
\hline Lauren type & & & & 0.508 & 0.476 \\
\hline Intestinal & 72 & 34 & 38 & & \\
\hline Diffuse & 56 & 30 & 26 & & \\
\hline Distant metastasis & & & & 20.802 & $<0.0001^{\mathrm{a}}$ \\
\hline No & 35 & 6 & 29 & & \\
\hline Yes & 93 & 58 & 35 & & \\
\hline $\begin{array}{l}\text { Degree of } \\
\text { differentiation }\end{array}$ & & & & 1.3474 & 0.2457 \\
\hline Highly differentiated & 38 & 16 & 22 & & \\
\hline $\begin{array}{l}\text { Moderately and } \\
\text { poorly differentiated }\end{array}$ & 90 & 48 & 42 & & \\
\hline HER-2 & & & & 0.1357 & 0.7126 \\
\hline++-+++ & 46 & 22 & 24 & & \\
\hline $0-+$ & 82 & 42 & 40 & & \\
\hline Ki-67 & & & & 0.011 & 0.918 \\
\hline$\geq 15 \%$ & 57 & 29 & 28 & & \\
\hline$<15 \%$ & 71 & 35 & 36 & & \\
\hline
\end{tabular}

MPV, mean platelet volume; HER-2, human epidermal growth factor

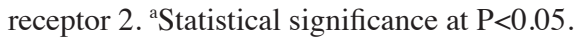

Table II. Association between the MPV baseline levels and chemotherapeutic efficacy.

\begin{tabular}{lcccc}
\hline $\begin{array}{l}\text { MPV } \\
\text { levels }\end{array}$ & $\begin{array}{c}\mathrm{PR}+\mathrm{SD} \\
(\mathrm{n}=81)\end{array}$ & $\begin{array}{c}\mathrm{PD} \\
(\mathrm{n}=47)\end{array}$ & $\chi^{2}$ test & P-value \\
\hline Low, $\mathrm{n}=60$ & 47 & 17 & 5.6822 & $0.0171^{\mathrm{a}}$ \\
High, $\mathrm{n}=60$ & 34 & 30 & & \\
\hline
\end{tabular}

MPV, mean platelet volume; PR, partial response; $\mathrm{SD}$, stable disease;

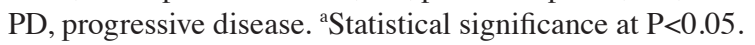

Follow up. The responses to chemoradiotherapy including complete remission, regression, stable disease, and disease progression, and overall and disease-free survival (DFS) were recorded. Survival time was measured from the date of chemo- 
Table III. Association between changes in the MPV level and chemotherapeutic efficacy.

\begin{tabular}{|c|c|c|c|c|c|}
\hline Pre-chemotherapy & Post-chemotherapy & $\mathrm{PR}+\mathrm{SD}(\mathrm{n}=81)$ & $\mathrm{PD}(\mathrm{n}=47)$ & $\chi^{2}$ test & P-value \\
\hline \multirow[t]{2}{*}{ Low (n=64) } & Low $(n=51)$ & 44 & 7 & 13.1977 & $0.0003^{\mathrm{a}}$ \\
\hline & $\operatorname{High}(n=13)$ & 5 & 8 & & \\
\hline \multirow[t]{2}{*}{ High $(n=64)$} & Low $(n=25)$ & 18 & 7 & 7.9426 & $0.0048^{\mathrm{a}}$ \\
\hline & High $(n=39)$ & 14 & 25 & & \\
\hline
\end{tabular}

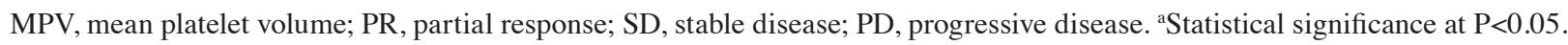
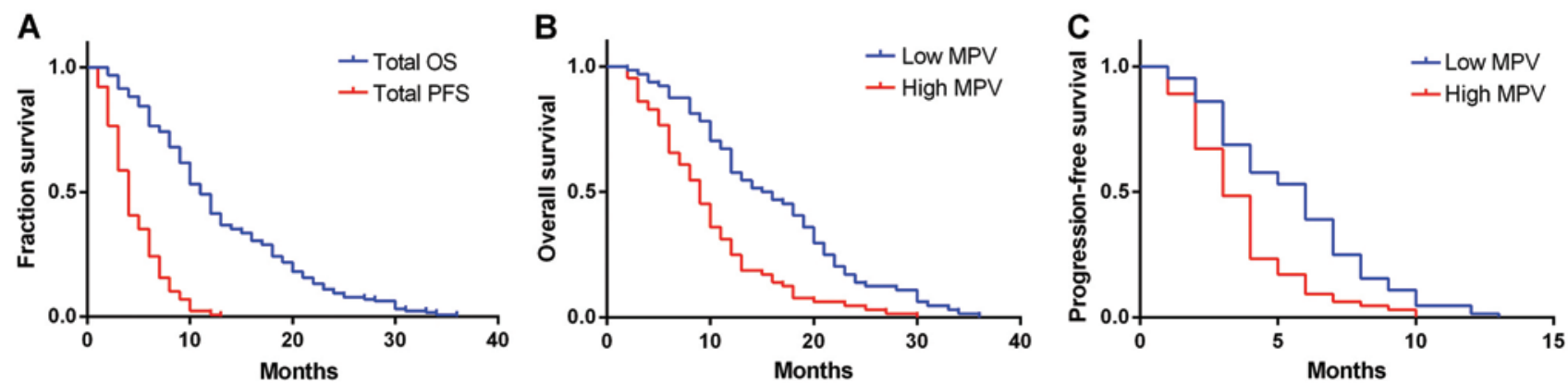

Figure 1. Association between the mean platelet volume (MPV) level and the outcomes. (A) Predicted probability of progression-free survival (PFS) and overall survival (OS). (B) OS and (C) PFS according to MPV.

radiotherapy until death or last clinical evaluation. Following first-line chemotherapy, disease progression after chemoradiotherapy was defined as lack of response to chemoradiotherapy. By contrast, stable disease, complete response or disease regression after chemoradiotherapy was defined as response to chemoradiotherapy. Patients were regularly followed up for 36 months. The prognostic analyses were performed based on progression-free survival (PFS) and overall survival (OS).

Statistical analysis. Statistical analyses were performed using SPSS 19.0 software (SPSS, Inc., Chicago, IL, USA). Multivariate Cox regression was performed for each outcome parameter, using a backwards elimination technique to derive a potentially suitable set of predictors. The association between the MPV level and clinicopathological characteristics or chemotherapeutic efficacy was examined and assessed by the $\chi^{2}$ tests. For analysis of survival data, Kaplan-Meier curves were constructed, and statistical analysis was carried out using the log-rank test. OS was defined as the time from the initiation of chemotherapy to the patient succumbing due to any cause. $\mathrm{P}<0.05$ was considered to indicate a statistically significant difference.

\section{Results}

Relationship between the baseline MPV level and clinicopathological characteristics. Patients were divided according to the median value of baseline MPV (MPV low: $<11.65$ or MPV high: $\geq 11.65$ ). The relationships between the baseline MPV level and clinicopathological characteristics were examined and assessed by the $\chi^{2}$ tests. The results showed that a low baseline MPV level was only correlated with reduced metastasis, but not with other clinicopathological characteristics (Table I).
Baseline MPV level predicts the chemotherapeutic efficacy. The association between the baseline MPV level and chemotherapeutic efficacy is provided in Table II. Patients with low baseline level of MPV had an improved response to chemotherapy, suggesting that the baseline MPV level did not predict chemotherapeutic efficacy.

Changes in MPV levels are associated with the chemotherapeutic efficacy. To define the association between changes in the MPV level with chemotherapeutic efficacy, blood samples were collected at the same time the CT evaluation was performed after first-line chemotherapy. The results showed that 51 patients with a low baseline MPV level, remained in this group after first-line chemotherapy (Table III). By contrast, 13 patients from this group were transferred into the high MPV level group. A total of 39 patients with a high baseline MPV level retained a high MPV level following first-line chemotherapy. By contrast, 25 patients with a high baseline MPV level were transferred into the low MPV level group. Patients remaining in or transferring into the low MPV level subgroup after first-line chemotherapy had an improved chemotherapy response, compared to those remaining in or transferring into the high level group.

MPV levels predict the outcomes. The median OS for all the patients was 11 months with a median PFS of 4 months (Fig. 1A). Follow up for survivors was 36 months. Kaplan-Meier plots showing the influence of MPV status on OS and PFS are shown in Fig. 1B and C. The median OS and PFS of the high MPV level group were 9 and 3 months, respectively, whereas that for the low MPV level group were 15.5 and 6 months, respectively. Significant differences 


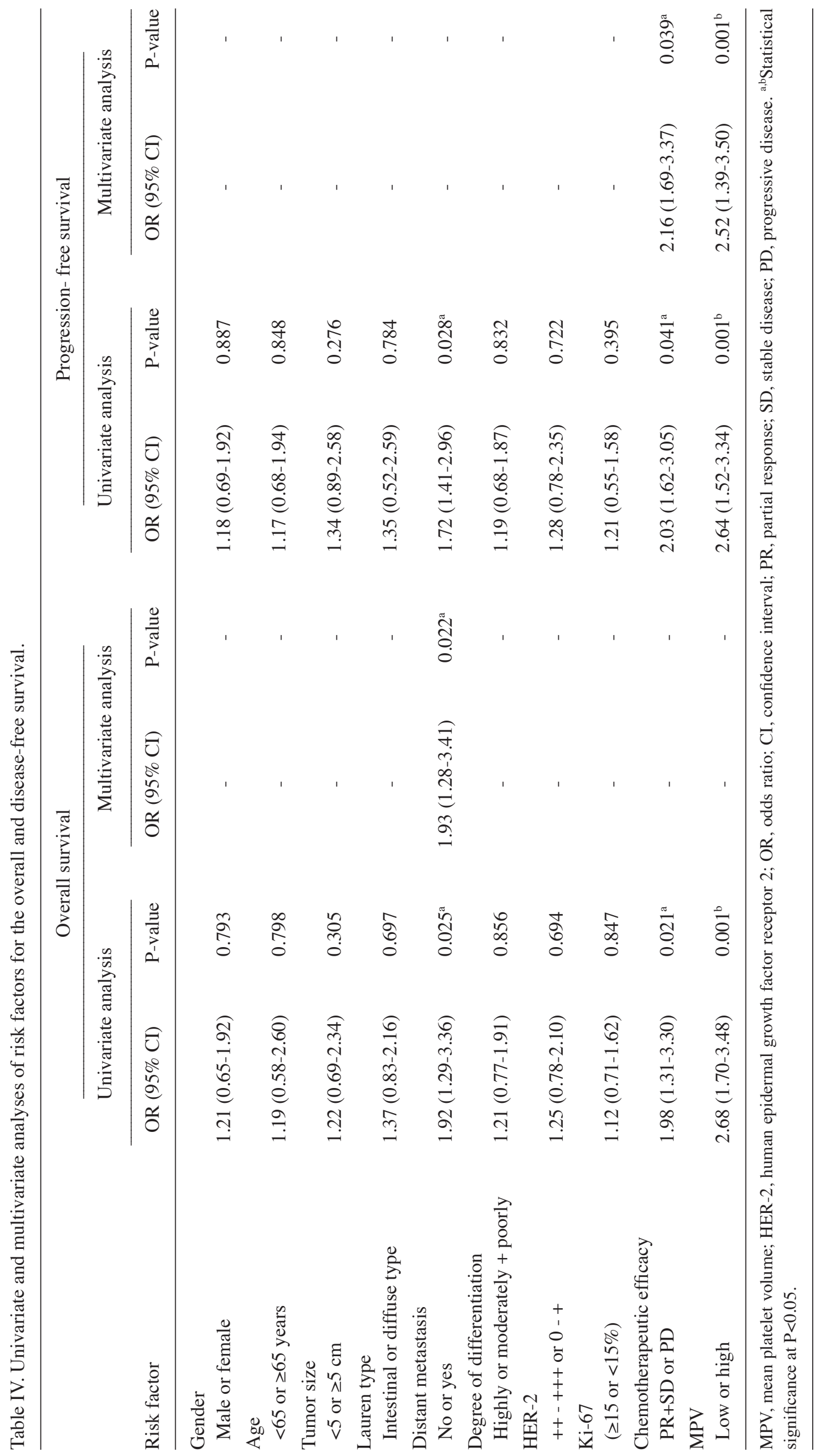


were identified between the OS and PFS of the two groups $(\mathrm{P}<0.001)$. Thus, the patients with a higher MPV level had decreased survival.

Univariate and multivariate analyses of risk factors for $O S$ and DFS. Univariate and multivariate analyses were performed to identify the risk factors associated with OS and PFS. As shown in Table IV, the univariate analysis revealed that 3 of the 10 risk factors affected OS and PFS, including distant metastasis, chemotherapeutic efficacy and MPV. The multivariate analysis confirmed that distant metastasis was the prognostic factor affecting OS. By contrast, the chemotherapeutic efficacy and MPV were prognostic factors affecting PFS.

\section{Discussion}

PLT activation is of paramount importance in the progression of malignancy. Previous findings have shown that the risk of cancer diagnosis is elevated after primary deep vein thrombosis or pulmonary embolism (17). Additionally, experimental and clinical data suggest that the activation of PLTs is a hallmark in the natural course of cancer, by promoting neoangiogenesis, degradation of the extracellular matrix, release of adhesion molecules, and growth factors, all of which are essential components for further tumor growth and metastatic spread (11).

Besides the impact that PLT activation has on cancer, an elevated PLT count in combination with other abnormal test results seems to be predictive for an underlying malignant disease (18), suggesting the potential of using PLT-associated factors as biomarkers for cancer diagnosis and treatment.

Evidence has shown that the larger PLTs are more reactive than the smaller ones and are more likely to aggregate, leading to thrombosis. Large PLTs (LPLTs) are independent risk factors for myocardial infarction, and PLT size is one predictor of recurrent myocardial infarction and death (19). A high level of MPV, a marker of PLT size, may indicate tendency towards thrombosis, and has been demonstrated in the case of myocardial infarction and cerebrovascular embolus (20). In cancer, an increase in the percentage of large PLTs has been observed, and because young, metabolically active PLTs appear in the circulation, this may lead to an increase in MPV (21). Recent findings have suggested that the MPV is a valuable biomarker for the diagnosis and follow up of various types of cancer (12-15).

The association between PLT and cancer may be linked by systemic inflammatory response (SIR) (22), which seems to play a critical role in the development and progression of various types of cancer by promoting cancer cell proliferation and survival, angiogenesis, tumor metastasis and impacting tumor response to systemic therapies (7). The mechanism involved in the effect on PLT by inflammation may be due to the release of pro-inflammatory cytokines, such as IL-1, IL-3 and IL-6, in many types of cancer. These cytokines have been proven to be able to promote the proliferation of megakaryocytes, resulting in PLT activation and aggregation, which potentially lead to the gradual establishment of thrombocytosis (11). This exact stimulation inevitably leads to an increased detection of more primitive types of circu- lating PLTs (11). This accelerated coagulation of PLTs may promote the metastasis of cancer cells. When covered with PLTs, cancer cells can overcome the stress in the bloodstream, including attacks by the immune system and physical factors (i.e., shear force and mechanical trauma due to passage through the microvasculature) $(8,23)$. Thus, the alliance of PLTs and cancer united by inflammation presents a positive feedback in the progression of malignancy.

Gastric cancer is usually located in the pyloric antrum and in the pylorus, but in $25 \%$ of cases in the body (corpus) and fundus of the stomach. Chronic inflammation of the stomach caused by Helicobacter pylori often leads to neoplastic transformation (21). Clinical and epidemiological studies have shown that gastric cancer is an inflammation-driven malignancy (24-26). Elevated serum concentrations of pro-inflammatory cytokines such as IL-6 were observed to be significantly higher in individuals with gastric cancer, as well as in patients with other inflammation-associated cancer types, such as colon and prostate cancers $(27,28)$. Therefore, we speculated that the elevated MPV level in gastric cancer patients may be due to a consequence of SIR. In patients with better chemotherapeutic efficacy, decreased MPV level may be due to remission of SIR, leading to a more favorable prognosis. By contrast, non-decreasing MPV may reflect persistent SIR and worse outcomes.

The results of the present study indicate that MPV may be used in the prediction of chemotherapy response and the follow up of gastric cancer. Considering the high gastric cancer morbidity and less developed economic conditions in China, this non-invasive, convenient and cost-effective biomarker may be beneficial in the treatment of gastric cancer.

\section{Acknowledgements}

The present study was supported by the National Natural Science Foundation of China (grant nos. 81472296, 81101867, $81272542,81200369$ and 81372443$)$, the China International Medical Foundation (grant no. CIMF F H001 057), the Special Foundation of Clinical Medicine of Jiangsu Provincial Bureau of Science and Technology (grant no. BL2014039), the Scientific Research Project of Jiangsu Provincial Bureau of Traditional Chinese Medicine (grant no. L213236), the Medical Scientific Research Project of Jiangsu Provincial Bureau of Health (grant no. Z201206), the Special Foundation of Wu Jieping Medical Foundation for Clinical Scientific Research (grant nos. 320.6753.1225 and 320.6750.12242), the Science and Education for Health Foundation of Suzhou for Youth (grant nos. SWKQ1003 and SWKQ1011), The Science and Technology Project Foundation of Suzhou (grant nos. SYS201112, SYSD2012137, SYS201335 and SYS201504), The Science and Technology Foundation of Suzhou Xiangcheng (grant nos. SZXC2012-70 and XJ201451) and a Project Founded by the Priority Academic Program Development of Jiangsu Higher Education Institutions.

\section{References}

1. Shen L, Shan YS, Hu HM, Price TJ, Sirohi B, Yeh KH, Yang YH, Sano T, Yang HK, Zhang X, et al: Management of gastric cancer in Asia: Resource-stratified guidelines. Lancet Oncol 14: e535-e547, 2013. 
2. Leung WK, Wu MS, Kakugawa Y, et al; Asia Pacific Working Group on Gastric Cancer: Screening for gastric cancer in Asia: current evidence and practice. Lancet Oncol 9: 279-287, 2008.

3. Wang WH, Huang JQ, Zheng GF, Lam SK, Karlberg J and Wong BC: Non-steroidal anti-inflammatory drug use and the risk of gastric cancer: a systematic review and meta-analysis. J Natl Cancer Inst 95: 1784-1791, 2003.

4. Kilınçalp S, Ekiz F, Başar O, Ayte MR, Coban S, Y1lmaz B, Altınbaş A, Başar N, Aktaş B, Tuna Y, et al: Mean platelet volume could be possible biomarker in early diagnosis and monitoring of gastric cancer. Platelets 25: 592-594, 2014.

5. Mroczko B, Wereszczyñska-Siemiatkowska U, Groblewska M, Lukaszewicz M, Szmitkowski M, Gryko M and Kedra B: The diagnostic value of hematopoietic cytokines measurement in the sera of gastric cancer and gastric ulcer patients. Clin Chim Acta 374: 165-167, 2006.

6. Mroczko B, Groblewska M, Łukaszewicz-Zajac M, Bandurski R, Kedra B and Szmitkowski M: Pre-treatment serum and plasma levels of matrix metalloproteinase 9 (MMP-9) and tissue inhibitor of matrix metalloproteinases 1 (TIMP-1) in gastric cancer patients. Clin Chem Lab Med 47: 1133-1139, 2009.

7. Kemal Y, Yucel I, Ekiz K, Demirag G, Yilmaz B, Teker F and Ozdemir M: Elevated serum neutrophil to lymphocyte and platelet to lymphocyte ratios could be useful in lung cancer diagnosis. Asian Pac J Cancer Prev 15: 2651-2654, 2014.

8. Lian L, Li W, Li ZY, Mao YX, Zhang YT, Zhao YM, Chen K, Duan WM and Tao M: Inhibition of MCF-7 breast cancer cell-induced platelet aggregation using a combination of antiplatelet drugs. Oncol Lett 5: 675-680, 2013.

9. Egan K, Crowley D, Smyth P, O'Toole S, Spillane C, Martin C, Gallagher M, Canney A, Norris L, Conlon N, et al: Platelet adhesion and degranulation induce pro-survival and pro-angiogenic signalling in ovarian cancer cells. PLoS One 6: e26125, 2011

10. Tsuruo T and Fujita N: Platelet aggregation in the formation of tumor metastasis. Proc Jpn Acad Ser B Phys Biol Sci 84: 189-198, 2008.

11. Seretis C, Seretis F, Lagoudianakis E, Politou M, Gemenetzis G and Salemis NS: Enhancing the accuracy of platelet to lymphocyte ratio after adjustment for large platelet count: a pilot study in breast cancer patients. Int J Surg Oncol 2012: 653608, 2012.

12. Cho SY, Yang JJ, You E, Kim BH, Shim J, Lee HJ, Lee WI, Suh JT and Park TS: Mean platelet volume/platelet count ratio in hepatocellular carcinoma. Platelets 24: 375-377, 2013.

13. Kemal Y, Demirag G, Ekiz K and Yucel I: Mean platelet volume could be a useful biomarker for monitoring epithelial ovarian cancer. J Obstet Gynaecol 34: 515-518, 2014.

14. Mutlu H, Berk V, Karaca H, Erden A, Aslan T and Akca Z: Treatment regimen with bevacizumab decreases mean platelet volume in patients with metastatic colon cancer. Clin Appl Thromb Hemost 18: 546-548, 2012.
15. Aksoy S, Kilickap S, Hayran M, Harputluoglu H, Koca E, Dede DS, Erman M and Turker A: Platelet size has diagnostic predictive value for bone marrow metastasis in patients with solid tumors. Int J Lab Hematol 30: 214-219, 2008.

16. Eisenhauer EA, Therasse P, Bogaerts J, Schwartz LH, Sargent D, Ford R, Dancey J, Arbuck S, Gwyther S, Mooney M, et al: New response evaluation criteria in solid tumours: revised RECIST guideline (version 1.1). Eur J Cancer 45: 228-247, 2009.

17. Bazou D, Santos-Martinez MJ, Medina C and Radomski MW: Elucidation of flow-mediated tumour cell-induced platelet aggregation using an ultrasound standing wave trap. Br J Pharmacol 162: 1577-1589, 2011.

18. Heras P, Hatzopoulos A, Kritikos N and Kritikos K: Platelet count and tumor progression in gastric cancer patients. Scand J Gastroenterol 45: 1005-1006, 2010.

19. Karagöz B, Bilgi O, Alacacioğlu A, Ozgün A, Sayan O, Erikçi AA and Kandemir EG: Mean platelet volume increase after tamoxifen, but not after anastrazole in adjuvant therapy of breast cancer. Med Oncol 27: 199-202, 2010.

20. Mutlu H, Artis TA, Erden A and Akca Z: Alteration in mean platelet volume and platicrit values in patients with cancer that developed thrombosis. Clin Appl Thromb Hemost 19: 331-333, 2013.

21. Matowicka-Karna J, Kamocki Z, Polińska B, Osada J and Kemona H: Platelets and inflammatory markers in patients with gastric cancer. Clin Dev Immunol 2013: 401623, 2013.

22. McMillan DC: Systemic inflammation, nutritional status and survival in patients with cancer. Curr Opin Clin Nutr Metab Care 12: 223-226, 2009.

23. Shou LM, Zhang QY, Li W, et al: Cantharidin and norcantharidin inhibit the ability of MCF-7 cells to adhere to platelets via protein kinase $\mathrm{C}$ pathway-dependent downregulation of $\alpha 2$ integrin. Oncol Rep 30: 1059-1066, 2013.

24. Ilhan N, Ilhan N, Ilhan Y, Akbulut H and Kucuksu M: C-reactive protein, procalcitonin, interleukin-6, vascular endothelial growth factor and oxidative metabolites in diagnosis of infection and staging in patients with gastric cancer. World J Gastroenterol 10: 1115-1120, 2004

25. Lochhead P and El-Omar EM: Gastric cancer. Br Med Bull 85: $87-100,2008$

26. Hussain SP and Harris CC: Inflammation and cancer: an ancient link with novel potentials. Int J Cancer 121: 2373-2380, 2007.

27. Galizia G, Lieto E, De Vita F, et al: Circulating levels of interleukin-10 and interleukin-6 in gastric and colon cancer patients before and after surgery: relationship with radicality and outcome. J Interferon Cytokine Res 22: 473-482, 2002.

28. Shariat SF, Andrews B, Kattan MW, Kim J, Wheeler TM and Slawin KM: Plasma levels of interleukin-6 and its soluble receptor are associated with prostate cancer progression and metastasis. Urology 58: 1008-1015, 2001. 